\title{
新型三元层状硼化物 $\mathrm{Cr}_{4} \mathrm{AlB}_{4}$ 的物相稳定性和力学行为分析
}

\author{
齐欣欣 ${ }^{1}$, 宋广平 ${ }^{1}$, 尹维龙 ${ }^{1}$, 王明福 ${ }^{2}$, 赫晓东 ${ }^{1}$, \\ 郑永挺 ${ }^{1}$, 王荣国 ${ }^{1}$, 柏跃䂞 ${ }^{1}$
}

(1. 哈尔滨工业大学 特种环境复合材料技术国家级重点实验室, 哈尔滨 150080; 2. 北京动力机械研究所, 北京 100074)

摘 要: $\mathrm{Cr}_{4} \mathrm{AlB}_{4}$ 是一种近期发现的三元层状嗍化物 MAB 相陶瓷。该材料可形成具有保护性的氧化膜, 在高温结构 材料领域有巨大应用潜力。本工作采用基于第一性原理的“线性优化法”和“键刚度”理论模型分别研究了 $\mathrm{Cr}_{4} \mathrm{AlB}_{4}$ 的 物相稳定性和力学行为。声子谱中没有虚频出现, 表明 $\mathrm{Cr}_{4} \mathrm{AlB}_{4}$ 具有本征稳定性。而与其它 $\mathrm{Cr}-\mathrm{Al}-\mathrm{B}$ 系内的竞争相 相比, $\mathrm{Cr}_{4} \mathrm{AlB}_{4}$ 具有最低的能量, 表明其在热力学上也是稳定的。采用“键刚度”模型对化学键刚度的定量计算显示, $\mathrm{Cr}_{4} \mathrm{AlB}_{4}$ 中 $\mathrm{Cr}$ 和 $\mathrm{B}$ 以及 $\mathrm{B}$ 和 $\mathrm{B}$ 原子之间形成了强共价键，而 $\mathrm{Cr}$ 和 $\mathrm{Al}$ 原子则形成相对较弱的 $\mathrm{Cr}-\mathrm{Al}(625 \mathrm{GPa})$ 和 B- $\mathrm{Al}(574 \mathrm{GPa})$ 键。 $\mathrm{Cr}_{4} \mathrm{AlB}_{4}$ 可以看成是由强共价键紧密连接在一起的 $\mathrm{Cr}-\mathrm{B}$ 结构单元, 被弱 $\mathrm{Cr}(\mathrm{B})-\mathrm{Al}$ 键分割而成的 层状结构, 与 $\mathrm{MAX}$ 相结构类似。 $\mathrm{Cr}_{4} \mathrm{AlB}_{4}$ 具有类似于 $\mathrm{MAX}$ 相的高损伤容限和断裂韧性。

关 键 词: MAB 相; 密度泛函理论; 物相稳定性; 力学性能

中图分类号: TQ134 文献标识码: A

\section{Analysis on Phase Stability and Mechanical Property of Newly-discovered Ternary Layered Boride $\mathrm{Cr}_{4} \mathrm{AlB}_{4}$}

\author{
QI Xin-Xin ${ }^{1}$, SONG Guang-Ping ${ }^{1}$, YIN Wei-Long ${ }^{1}$, WANG Ming-Fu ${ }^{2}$, HE Xiao-Dong ${ }^{1}$, \\ ZHENG Yong-Ting ${ }^{1}$, WANG Rong-Guo ${ }^{1}$, BAI Yue-Lei ${ }^{1}$
}

(1. National Key Laboratory of Science and Technology on Advanced Composites in Special Environments, Harbin Institute of Technology, Harbin 150080, China; 2. Beijing Power Machinery Research Institute, Beijing 100074, China)

\begin{abstract}
As newly-discovered member of the MAB phases, $\mathrm{Cr}_{4} \mathrm{AlB}_{4}$ has much potential for high-temperature structural applications due to possible formation of a protective oxide scale. By use of "linear optimization procedure" and theoretical model of "bond stiffness" based on first-principle calculations, the phase stability and mechanical behavior of $\mathrm{Cr}_{4} \mathrm{AlB}_{4}$ were investigated. No imaginary frequencies in phonon dispersion indicate the intrinsic stability. The lower energy as compared with the set of other competing phases also shows the thermodynamic stability. Based on the quantificationally calculated bond stiffness by use of the model of "bond stiffness", strong covalent bonding is present between $\mathrm{Cr}$ and $\mathrm{B}$ atoms as well as $\mathrm{B}$ and $\mathrm{B}$ atoms, while the $\mathrm{Cr}-\mathrm{Al}$ (625 GPa) and $\mathrm{B}-\mathrm{Al}$ (574 GPa) bond is relatively weak. It follows that $\mathrm{Cr}_{4} \mathrm{AlB}_{4}$ can be described as layered structure of strong covalently bonded $\mathrm{Cr}-\mathrm{B}$ blocks interleaved by $\mathrm{Al}$ atomic planes where the bonding is relatively weak, similar to the well-known MAX phases, which demonstrates the similar damage tolerance and fracture toughness of $\mathrm{Cr}_{4} \mathrm{AlB}_{4}$ with the MAX phases.
\end{abstract}

Key words: MAB phases; density functional theory; phase stability; mechanical property

收稿日期: 2019-04-17; 收到修改稿日期：2019-05-14

基金项目: 国家自然科学基金(51972080); 黑龙江省自然科学基金重点项目(ZD2017011); 中国博士后科学基金(2017T100230) National Natural Science Foundation of China (51972080); Natural Science Foundation of Heilongjiang Province of China (ZD2017011); China Postdoctoral Science Foundation (2017T100230)

作者简介: 齐欣欣(1995-), 博士研究生. E-mail: 16S118138@stu.hit.edu.cn QI Xin-Xin(1995-), PhD candidate. E-mail: 16S118138@stu.hit.edu.cn

通讯作者：柏跃否，副教授. E-mail: baiyl@hit.edu.cn BAI Yue-Lei, associate professor. E-mail: baiyl@hit.edu.cn 
超高温陶瓷(Ultra-High Temperature Ceramics, UHTCs)凭借优良的高温性能在航空航天领域具有 广阔的应用前景, 其中二元硼化物更是具备了较好 的综合性能 ${ }^{[1]}$ 。然而, 二元硼化物的本征脆性和较差 的抗氧化性是限制其实现应用的主要障碍。近二十 年来, 在二元碳化物、氮化物中插入主族原子形成 的 $\mathrm{M}_{n+1} \mathrm{AX}_{n}$ 相化合物( $\mathrm{M}$ 为过渡金属元素, $\mathrm{A}$ 是 IIIA 或 IVA 主族元素, $\mathrm{X}$ 是 $\mathrm{C}$ 或 $\mathrm{N}, n=1-3)$ 由于具备高断 裂韧性、高损伤容限、良好的抗热震和抗氧化性能 而备受人们关注 ${ }^{[2]}$ 。受 MAX 相的启发, 在二元硼化 物中插入单层或双层主族原子如 $\mathrm{Al}$ 原子也可形成 被称为“MAB 相”的三元层状过渡金属嗍化物 ${ }^{[3]}$ 。

大部分 MAB 相陶瓷早在 20 世纪 60 年代就已 经被发现 ${ }^{[4-5]}$, 但直到 2013 年细致的结构和性能表 征才陆续开展 ${ }^{[6]}$ 。2015 年 Ade 等 $^{[3]}$ 合成出一系列的 $\mathrm{MAB}$ 相化合物单晶并对其结构和硬度进行了研究。 而最近的研究工作显示 $\mathrm{MoAlB}^{[7]}$ 和 $\mathrm{Fe}_{2} \mathrm{AlB}_{2}{ }^{[8]}$ (典型 的 MAB 相化合物)具有较高的断裂韧性和损伤容限 以及良好的抗氧化性能, 这对其潜在的结构应用极 为重要。Cr-Al-B 系 MAB 相化合物在氧化过程中形 成致密的 $\mathrm{Cr}_{2} \mathrm{O}_{3}$ 或 $\mathrm{Al}_{2} \mathrm{O}_{3}$ 氧化膜, 具有良好的抗氧化 性能，因而受到了广泛关注 ${ }^{[9-11]}$ 。具有不同晶体结构 的 $\mathrm{Cr}_{2} \mathrm{AlB}_{2} 、 \mathrm{Cr}_{3} \mathrm{AlB}_{4}$ 和 $\mathrm{Cr}_{4} \mathrm{AlB}_{6}$ 很早就被发现 ${ }^{[12]}$, 该 体系也是目前发现 MAB 相材料最多的体系。最近, 一种新型的 $\mathrm{MAB}$ 相化合物 $\mathrm{Cr}_{4} \mathrm{AlB}_{4}$ 在该体系中被 发现 ${ }^{[13]}$, 其结构不同于之前发现的任何一种 MAB 相化合物。第一性原理研究工作预测了它的晶体结 构、电子结构、化学键合、理论强度和以及热膨胀 行为等 ${ }^{[14]}$ 。

对结构陶瓷来说, 力学性能特别是损伤容限和 断裂韧性对其工程应用极其关键。而晶体结构对材 料的宏观力学行为具有重要影响。一般认为 MAX 相材料中的高损伤容限和高断裂韧性即来源于其层 状结构和层间的弱化学键结合 ${ }^{[2]}$ 。由于目前尚不能 制备高纯块体 $\mathrm{Cr}_{4} \mathrm{AlB}_{4}$ 材料而无法对其力学行为进 行实验表征, 第一性原理方法具有成本低、效率高、 可精确计算材料基态性质的优点, 已被广泛应用于 材料性能表征 ${ }^{[15-16]}$, 本工作采用第一性原理方法对 $\mathrm{Cr}_{4} \mathrm{AlB}_{4}$ 的物相稳定性、压缩和力学行为进行分析, 为深入理解和认识晶体结构对 MAB 相宏观行为的 影响和进一步实验研究提供理论指导。

\section{1 计算参数及模型设置}

\section{1 通用参数}

本研究利用 $\mathrm{VASP}^{[17]}$ 软件包计算 $\mathrm{Cr}_{4} \mathrm{AlB}_{4}$ 的平
衡晶格常数和其它性能。电子和离子之间的作用选 择 PAW 方法描述，其中 $\mathrm{Cr}$ 原子把 $\mathrm{p}$ 电子作为半芯 态来处理, $\mathrm{Cr} 、 \mathrm{Al}$ 和 $\mathrm{B}$ 原子选取的价电子分别为 $3 p^{6} 3 d^{5} 4 s^{1} 、 3 s^{2} 3 p^{1}$ 和 $2 s^{2} 2 p^{1}$ 。先选取不同的交换关联 泛函包括广义梯度近似 $\left(\mathrm{GGA}^{[18]}-\mathrm{PBE}, \mathrm{RPBE}^{[19]}\right.$, PW91 ${ }^{[20]}$ )和局域密度近似(LDA)来寻找与实验值最 接近的结果, 对其它参数选取计算精度最好的交换 关联函数进行计算。平面波矢的截断能选取为 $450 \mathrm{eV}$, 布里渊区 $\mathbf{k}$ 点积分采用 Monkhorst-Pack 方法, 网格 设定为 $13 \times 2 \times 13$ 。经测试, 该截断能和 $\mathbf{k}$ 点网格使 总能变化小于 $1 \mathrm{meV} / \mathrm{atom}$ 。计算态密度时, $\mathbf{k}$ 点网格 加密设定为 $39 \times 6 \times 39$ 。结构优化所用的收敛判据为: 离子弛豫过程中的总能量变化小于 $10^{-5} \mathrm{eV}$, 电子弛 豫过程中总能变化小于 $10^{-6} \mathrm{eV}$, 作用于原子的力小 于 $0.01 \mathrm{eV} / \mathrm{nm}$ 。

力常数矩阵和声子振动频率利用基于有限位移 法的 PHONOPY 软件包计算得到, 而其中的电子结 构计算则由 VASP 完成。具体方法为: 在 $\mathrm{Cr}_{4} \mathrm{AlB}_{4}$ 晶胞中每个非等同原子上施加一套独立的小位移, 然后通过电子结构计算得到原子间的 HellmannFeynman 作用力, 进一步得到原子间的力常数矩 阵。本文构造了一个 $2 \times 1 \times 2$ 超胞并采用 $7 \times 4 \times 7$ 的 $\mathbf{k}$ 点网格用于计算扰动原子产生的力。收玫条件是总 能量的变化小于 $10^{-8} \mathrm{eV}$

\section{2 物相稳定性}

本研究主要从本征稳定性和热力学稳定性角度 研究 $\mathrm{Cr}_{4} \mathrm{AlB}_{4}$ 的物相稳定性。本征稳定性指的是结 构的吉布斯自由能对于小变形处于一个极小值附 近。这是任何一个化合物或结构稳定存在的必要条 件。在实际研究工作中, 可以通过考察晶格动力学 (声子)行为来加以确定; 稳定结构中不应该出现负 频率的振动模态, 因为这意味着所研究的结构并不 处于局域能量极小值。

热力学稳定性是指与体系中其它竞争相或者竞 争相(结构)组合相比应处于能量最低状态。Dahlqvist 等 ${ }^{[21]}$ 提出了一种研究 MAX 相稳定性的方法: 它利 用线性规划法来寻找某一给定元素组成 $b^{\mathrm{M}}, b^{\mathrm{A}}$ 和 $b^{\mathrm{B}}$ 结构中最稳定的竞争相组合(能量最低)。本文用该 方法研究 MAB 相的稳定性, 可用下式表示:

$$
\min E_{\text {comp }}\left(b^{\mathrm{M}}, b^{\mathrm{A}}, b^{B}\right)=\sum_{i=1}^{n} x_{i} E_{i}
$$

其中, $x_{i}$ 和 $E_{i}$ 分别是化合物 $i$ 的数量和对应的能量, 并且受线性规划最小值约束。考虑到质量守恒，则有:

$$
x_{i} \geqslant 0, b^{\mathrm{M}}=\sum_{i=1}^{n} x_{i}^{\mathrm{M}}, b^{\mathrm{A}}=\sum_{i=1}^{n} x_{i}^{\mathrm{A}}, b^{\mathrm{B}}=\sum_{i=1}^{n} x_{i}^{\mathrm{B}}
$$


对 $\mathrm{Cr}_{4} \mathrm{AlB}_{4}, b^{\mathrm{M}}=4, b^{\mathrm{A}}=1, b^{\mathrm{B}}=4$ 。因此，任一 $\mathrm{MAB}$ 相相对于所确定的最具竞争性相组成的形成焓可根 据下式计算:

$$
\Delta H_{\text {comp }}=E_{\mathrm{MAB}}-\min E_{\text {comp }}\left(b^{\mathrm{M}}, b^{\mathrm{A}}, b^{\mathrm{X}}\right)
$$

根据形成焓 $\Delta H_{\text {comp }}$ 的正负来评估所研究化合物 的热力学稳定性: 若 $\Delta H_{\mathrm{comp}}$ 为正, 则所研究的 $\mathrm{MAB}$ 相不稳定; 反之, 则稳定。

\section{3 “键刚度”模型}

假设化学键在静水压力作用下的变形规律类似 于弹簧而满足胡克定律, 其中刚度用于定量表征化 学键强度。根据优化后的晶格参数和坐标可以很方 便地计算原子间距离(键长, $d$ )与静水压力 $P$ 之间的 关系。由于键强随原子间距离的增加而变弱, 所以 相对键长 $d / d_{0}$ ( $d_{0}$ 为 $0 \mathrm{GPa}$ 的键长度) 随 $P$ 的变化用 二阶多项式拟合, 该曲线的斜率定义为 $1 / k$, 其中 $k$ 是化学键刚度 ${ }^{[22]}$.

$$
\begin{gathered}
d / d_{0}=\mathrm{C}_{0}+\mathrm{C}_{1} P+\mathrm{C}_{2} P^{2} \\
k=\left|\frac{\mathrm{d}\left(d / d_{0}\right)}{\mathrm{d} P}\right|^{-1}=\left|\mathrm{C}_{1}+2 \mathrm{C}_{2} P\right|^{-1}
\end{gathered}
$$

其中 $C_{i}(i=0,1,2)$ 是二阶多项式拟合系数。

\section{4 二阶弹性常数和弹性模量}

二阶弹性常数 $c_{\mathrm{ij}}$ 是通过对晶格进行六次有限变 形后根据应力应变关系拟合来确定的。默认步长为 $0.0015 \mathrm{~nm}$ 。对于每个应变分量, 施加两个正 $(0.015$, $0.003)$ 和两个负 $(-0.015,-0.003)$ 应变, 然后根据计算 应力随应变的函数线性拟合确定弹性刚度。工程弹 性常数包括体积模量 $(B)$, 剪切模量 $(G)$, 杨氏模量 $(E)$ 和泊松比 $(\mu)$ 根据 Voigt 近似从二阶弹性常数确 定 ${ }^{[23]}$ 。德拜温度 $\left(\theta_{\mathrm{D}}\right)$ 的计算方法详见文献[24]。

\section{2 结果与讨论}

\section{1 晶体结构}

采用不同交换关联泛函(GGA-PBE、GGA-RPBE、
GGA-PW91、LDA)计算获得的平衡晶格参数如表 1 所示。LDA 所算出的晶格常数最小, 且明显小于实 验值。需要指出的是, LDA 通常低估晶格常数。GGA 的计算结果与实验值较为接近, 但都小于实验值, 这与对 MoAlB 的计算结果并不相同 ${ }^{[25]}$, 其中 RPEB 的计算结果与实验值最为接近(误差小于 $1 \%$ ), 故在 之后的计算中均采用 RPBE。根据表 1 还可以发现 随着 $\mathrm{Al}$ 层插入到 $\mathrm{Cr}-\mathrm{B}$ 结构中, 晶格常数 $a$ 和 $c$ 由 $\mathrm{CrB}$ 的 0.2924 和 $0.2911 \mathrm{~nm}$ 增加到 0.29334 和 $0.29551 \mathrm{~nm}$, 并且 $\mathrm{Cr}_{4} \mathrm{AlB}_{4}$ 与其它 Cr-Al-B 系 $\mathrm{MAB}$ 相具有相近的密度 ${ }^{[26]}$ 。实际上, 之前的结果也显示 了 $\mathrm{Cr}-\mathrm{B}$ 层片数的增加并不显著提高 $\mathrm{Cr}-\mathrm{Al}-\mathrm{B}$ 系 $\mathrm{MAB}$ 相的密度 ${ }^{[26]}$ 。

图 1 为 $\mathrm{Cr}_{4} \mathrm{AlB}_{4}$ 及 $\mathrm{CrB}$ 的晶体结构, 图中显示 $\mathrm{Cr}-\mathrm{B}$ 层沿 $b$ 轴方向被 $\mathrm{Al}$ 单原子层分离。换句话说, $\mathrm{Cr}_{4} \mathrm{AlB}_{4}$ 看作二元硼化物沿 $b$ 轴方向插入单层铝原 子而形成的。 $\mathrm{Cr}_{4} \mathrm{AlB}_{4}$ 还可以描述为类似于 $\mathrm{MAX}$ 相 的层状结构: 结合力较弱 $\mathrm{Cr}-\mathrm{Al}$ 原子层与强共价键 结合的 $\mathrm{Cr}-\mathrm{B}$ 层交错堆叠。相邻原子之间的键长列于 表 2(在图 1 中标记的相关原子), 并对二元化合物 $\mathrm{CrB}$ 的相应数据进行了比较。对于 $\mathrm{Cr}_{4} \mathrm{AlB}_{4}, \mathrm{Cr}-\mathrm{B}$ 层 与 $\mathrm{CrB}$ 具有类似的构型, $\mathrm{B}-\mathrm{B}$ 链与 $\mathrm{CrB}$ 相比有轻微 的扁平化, 在 $\mathrm{Cr}_{4} \mathrm{AlB}_{4}$ 中 $\mathrm{B} 1-\mathrm{B} 2$ 和 $\mathrm{Cr} 1-\mathrm{B} 2$ 键长分别 为 0.1771 和 $0.2180 \mathrm{~nm}(\mathrm{CrB}$ 中分别为 0.17841 和 $0.2212 \mathrm{~nm})$ 。而两层 $\mathrm{Cr}-\mathrm{B}$ 之间的距离明显增加, $\mathrm{Cr} 2-\mathrm{B} 3$ 的键长为 $0.2288 \mathrm{~nm}_{\circ} \mathrm{Cr}_{4} \mathrm{AlB}_{4}$ 与其它已知的 $\mathrm{Cr}-\mathrm{Al}-\mathrm{B}$ 系 MAB 相明显不同的一点是其它 MAB 相 $\mathrm{Cr}$ 和 $\mathrm{B}$ 原子的构型取决于 $\mathrm{Cr}-\mathrm{B}$ 层中的位置: 在边 缘处, 构型与 $\mathrm{CrB}$ 相似, 在 $\mathrm{Cr}-\mathrm{B}$ 层中, 原子采用 $\mathrm{CrB}_{2}$ 的结构, $\mathrm{B}$ 原子形成平面的六方环 ${ }^{[26]}$ 。

\section{2 物相稳定性分析}

$\mathrm{Cr}_{4} \mathrm{AlB}_{4}$ 的声子色散曲线和态密度如图 2 所示。 在声子谱中没有虚频出现, 这意味着 $\mathrm{Cr}_{4} \mathrm{AlB}_{4}$ 的吉 布斯自由能对于小变形处于一个极小值附近, 表明 其是本征稳定的。如图 2 所示, $14 \mathrm{THz}$ 以上的高频

\begin{tabular}{|c|c|c|c|c|c|c|c|c|c|c|c|c|c|c|c|}
\hline & & & \multicolumn{4}{|c|}{ Lattice parameters } & \multicolumn{5}{|c|}{ Internal coordinates } & \multirow{2}{*}{$\begin{array}{l}\text { Density } \\
/\left(\mathrm{g} \cdot \mathrm{cm}^{-3}\right)\end{array}$} & \multirow{2}{*}{$\begin{array}{c}\text { DOS at } E_{\mathrm{f}} \\
/\left(\mathrm{States} \cdot \mathrm{eV}^{-1}\right. \\
\left.\text { Unit cell }^{-1}\right)\end{array}$} & \multirow{2}{*}{$\begin{array}{c}\text { Bulk } \\
\text { modulus } \\
/ \mathrm{GPa}\end{array}$} & \multirow{2}{*}{$\begin{array}{c}\text { Pressure } \\
\text { deriva- } \\
\text { tive }\end{array}$} \\
\hline & & & $a / \mathrm{nm}$ & $b / \mathrm{nm}$ & $c / \mathrm{nm}$ & $V / \mathrm{nm}^{3}$ & $y_{\mathrm{Cr} 1}$ & $y_{\mathrm{Cr} 2}$ & $y_{\mathrm{Al}}$ & $y_{\mathrm{B} 1}$ & $y_{\mathrm{B} 2}$ & & & & \\
\hline & LDA & & 0.28766 & 1.8578 & 0.28867 & 0.15427 & - & - & - & - & - & - & - & - & - \\
\hline & & PBE & 0.29312 & 1.8923 & 0.29512 & 0.16370 & - & - & - & - & - & - & & - & - \\
\hline \multirow[t]{3}{*}{$\mathrm{Cr}_{4} \mathrm{AlB}_{4}$} & GGA & RPBE & 0.29334 & 1.8963 & 0.29551 & 0.16439 & 0.2926 & 0.5875 & 0.5 & 0.3803 & 0.6720 & 5.64 & 7.031 & 234.16 & 4.55 \\
\hline & & PW91 & 0.29197 & 1.8863 & 0.29435 & 0.16212 & - & - & - & - & - & - & - & - & - \\
\hline & $\operatorname{Exp}^{[13]}$ & & 0.29343 & 1.8891 & 0.29733 & 0.16481 & 0.2936 & 0.5859 & 0.5 & 0.3839 & 0.6646 & - & - & - & - \\
\hline $\mathrm{CrB}$ & $\mathrm{Cal}^{[27]}$ & & 0.2924 & 0.7836 & 0.2911 & 0.06670 & - & - & - & - & - & 6.25 & - & - & - \\
\hline
\end{tabular}

表 $1 \mathrm{Cr}_{4} \mathrm{AlB}_{4}$ 及 $\mathrm{CrB}$ 晶格参数及基本属性

Table 1 Lattice parameters and some basic property of $\mathrm{Cr}_{4} \mathrm{AlB}_{4}$ and $\mathrm{CrB}$ 
(a)

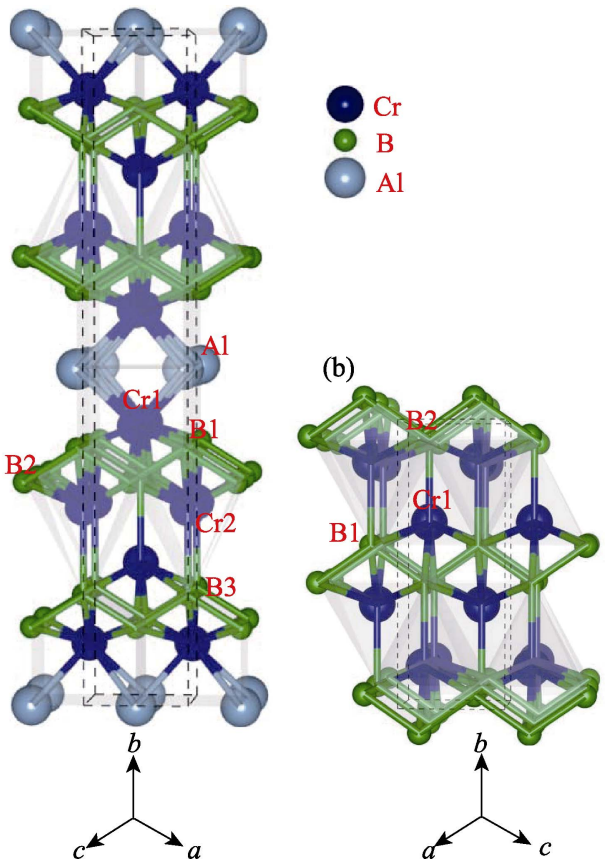

图 $1 \mathrm{Cr}_{4} \mathrm{AlB}_{4}(\mathrm{a})$ 和 $\mathrm{CrB}(\mathrm{b})$ 的晶体结构

Fig. 1 Crystal structure of $\mathrm{Cr}_{4} \mathrm{AlB}_{4}$ (a) and $\mathrm{CrB}$ (b) $\mathrm{Cr}_{4} \mathrm{AlB}_{4}$ can be regarded as $\mathrm{CrB}$ with $\mathrm{Al}$ monolayer inserted along the $\mathrm{b}$ axis
声子态主要是由 $\mathrm{B}$ 原子的振动引起的, 而低频的晶 格振动则主要归因于 $\mathrm{Cr}$ 和 $\mathrm{Al}$ 原子。 $\mathrm{B}$ 原子的高频 率是由于它的强化学键合和低原子质量。 $\mathrm{Cr}$ 原子由 于其高质量导致其具有较低的频率，而相反地，由 于具有较弱的键合(尽管它的质量较低), $\mathrm{Al}$ 原子占 据低频的相似范围。这与其它 Cr-Al-B 系 MAB 相 的计算结果相似 ${ }^{[26]}$ 。

通过应用 Dahlqvist 等 ${ }^{[21]}$ 的方法来评估 $\mathrm{Cr}_{4} \mathrm{AlB}_{4}$ 的热力学稳定性, 结果如表 3 所示。可以发现, $\mathrm{Cr}_{4} \mathrm{AlB}_{4}$ 的 $\Delta H_{\text {comp }}$ 小于 0 , 证明 $\mathrm{Cr}_{4} \mathrm{AlB}_{4}$ 是热力学稳 定的。值得注意的是, $\mathrm{Cr}_{4} \mathrm{AlB}_{4}$ 的 $\Delta H_{\text {comp }}$ 小于 $\mathrm{Cr}_{2} \mathrm{AlB}_{2}$ 的 $\Delta H_{\text {comp }}$, 说明 $\mathrm{Cr}_{4} \mathrm{AlB}_{4}$ 具有更高的热力学稳定性, 这也解释了在合成实验时, $\mathrm{Cr}_{2} \mathrm{AlB}_{2}$ 的合成温度低于 $\mathrm{Cr}_{4} \mathrm{AlB}_{4}{ }^{[13]}$ 。

\section{3 可压缩行为与键刚度}

通过研究材料的可压缩行为可以探究材料的各 向异性、化学键合和高压稳定性等。因此, MAX 相 的可压缩性一直是重要的实验 ${ }^{[28]}$ 和理论研究 ${ }^{[29]}$ 方 向。基于密度泛函理论的第一性原理研究方法被广

表 $2 \mathrm{Cr}_{4} \mathrm{AlB}_{4} 、 \mathrm{Cr}_{2} \mathrm{AlB}_{2}$ 以及二元硼化物 $\mathrm{CrB}$ 的键长和键刚度

Table 2 Bond length and bond stiffness in $\mathrm{Cr}_{4} \mathrm{AlB}_{4}$, and $\mathrm{Cr}_{2} \mathrm{AlB}_{2}$ as well as binary borides $\mathrm{CrB}$

\begin{tabular}{|c|c|c|c|c|c|c|c|}
\hline & \multicolumn{2}{|c|}{$\mathrm{Cr}_{4} \mathrm{AlB}_{4}$} & \multicolumn{2}{|c|}{$\mathrm{Cr}_{2} \mathrm{AlB}_{2}$} & \multicolumn{3}{|c|}{$\mathrm{CrB}$} \\
\hline & $d / \mathrm{nm}$ & $k / \mathrm{GPa}$ & $d / \mathrm{nm}^{[3]}$ & $k / \mathrm{GPa}^{[26]}$ & $d / \mathrm{nm}$ & $d / \mathrm{nm}^{[27]}$ & $k / \mathrm{GPa}$ \\
\hline $\mathrm{Cr} 1-\mathrm{Al}$ & 0.2662 & 625 & 0.26443 & 617 & - & - & - \\
\hline $\mathrm{Cr} 1-\mathrm{B} 1$ & 0.2169 & 885 & 0.22014 & 813 & 0.21712 & 0.2158 & 917 \\
\hline $\mathrm{Cr} 1-\mathrm{B} 2$ & 0.2180 & 1190 & 0.21876 & 1149 & 0.2212 & 0.2200 & 1123 \\
\hline $\mathrm{Cr} 2-\mathrm{B} 1$ & 0.2224 & 1074 & - & - & - & - & - \\
\hline $\mathrm{Cr} 2-\mathrm{B} 2$ & 0.2187 & 840 & - & - & - & - & - \\
\hline Cr2-B3 & 0.2288 & 826 & - & - & - & - & - \\
\hline B1-B2 & 0.1771 & 1123 & 0.17616 & 1099 & 0.17841 & 0.1722 & 1149 \\
\hline Al-B1 & 0.2269 & 574 & 0.22765 & 571 & - & - & - \\
\hline
\end{tabular}
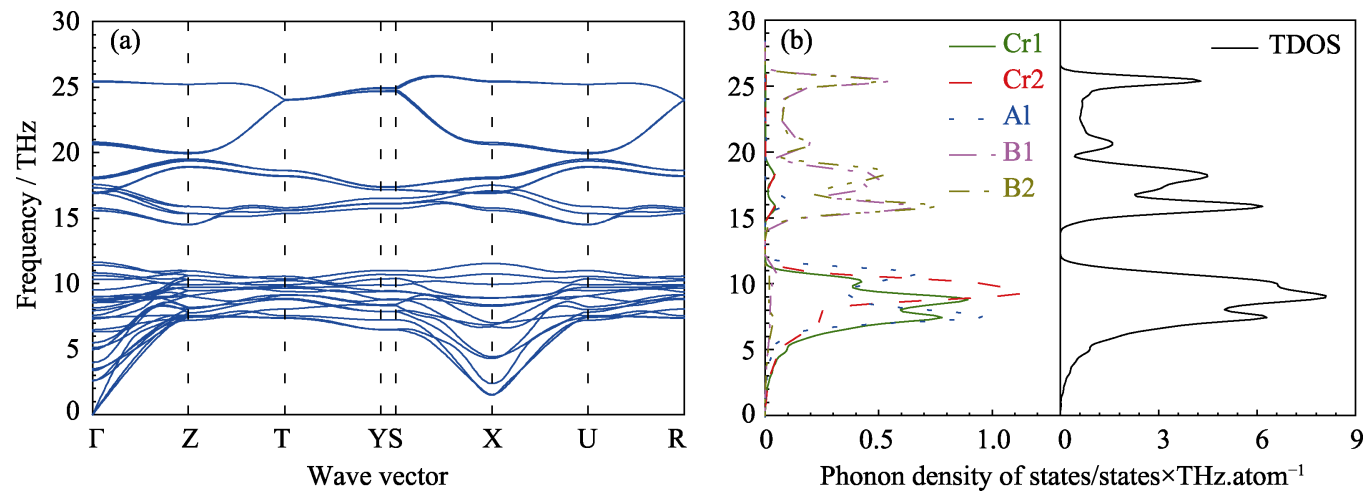

图 $2 \mathrm{Cr}_{4} \mathrm{AlB}_{4}$ 沿高对称点方向的声子色散曲线(a)和态密度(b)

Fig. 2 Phonon dispersions (a) and density of state (b) of $\mathrm{Cr}_{4} \mathrm{AlB}_{4}$ along the high symmetry directions 
表 $3 \mathrm{Cr}_{4} \mathrm{AlB}_{4}$ 的形成焓 $\Delta \boldsymbol{H}_{\mathrm{comp}}, \Delta \boldsymbol{H}_{\mathrm{comp}}<0$ 的相以粗体表示

Table 3 Formation enthalpy $\Delta H_{\text {comp }}$ for $\mathrm{Cr}_{4} \mathrm{AlB}_{4}$, phases with $\Delta H_{\text {comp }}<0$ are in bold

\begin{tabular}{cccc}
\hline Included phases & Compound & Most competing phases & $\Delta H_{\text {comp }} /(\mathrm{eV} \cdot$ atom \\
\hline $\mathrm{CrB}, \mathrm{CrB}_{2}, \mathrm{CrB}_{4}, \mathrm{Cr}_{2} \mathrm{~B}, \mathrm{Cr}_{5} \mathrm{~B}_{3}, \mathrm{Cr}_{3} \mathrm{~B}_{4}, \mathrm{Cr}_{2} \mathrm{~B}_{3}, \mathrm{Cr}_{2} \mathrm{Al}, \mathrm{Cr}_{5} \mathrm{Al}_{8}$, & $\mathrm{Cr}_{4} \mathrm{AlB}_{4}$ & $2 \mathrm{CrB}+\mathrm{Cr}_{2} \mathrm{AlB} \mathrm{B}_{2}$ & -0.2207 \\
$\mathrm{Cr}_{7} \mathrm{Al}_{45}, \mathrm{AlB}_{2}, \mathrm{AlB}_{12}, \mathrm{Cr}_{2} \mathrm{AlB}_{2}, \mathrm{Cr}_{3} \mathrm{AlB}_{4}, \mathrm{Cr}_{4} \mathrm{AlB}_{6}$ & $\mathrm{Cr}_{2} \mathrm{AlB}_{2}{ }^{[26]}$ & $2 \mathrm{CrB}+\mathrm{Al}$ & -0.0461 \\
\hline
\end{tabular}

泛应用于研究高压下的体积模量、化学键合和相稳 定性。图 3(a)显示了 $\mathrm{Cr}_{4} \mathrm{AlB}_{4}$ 的晶胞体积随静水压 力的变化关系, 该曲线可用 Birch-Murnaghan 方程 ${ }^{[30]}$ (状态方程)表示:

$$
\begin{gathered}
P(V)=\frac{3 B_{0}}{2}\left[\left(\frac{V}{V_{0}}\right)^{-7 / 3}-\left(\frac{V}{V_{0}}\right)^{-5 / 3}\right] \\
\left\{1+\frac{3}{4}\left(B_{0}^{\prime}-4\right)\left[\left(\frac{V}{V_{0}}\right)^{-2 / 3}-1\right]\right\}
\end{gathered}
$$

式中, $V$ 为晶胞体积, $B_{0}$ 为体积模量, $B^{\prime}{ }_{0}$ 为压力导数, $V_{0}$ 为 $0 \mathrm{GPa}$ 时的晶胞体积。通过拟合该方程可获得 $\mathrm{Cr}_{4} \mathrm{AlB}_{4}$ 的 $B_{0}$ 及 $B_{0}^{\prime}$ 分别为 $234 \mathrm{GPa}$ 和 4.55 。

表 2 列出了采用“键刚度”模型计算出的在 $0 \mathrm{GPa}$ 时化学键刚度。 $\mathrm{Cr}_{4} \mathrm{AlB}_{4}$ 中刚度最低的键为 $\mathrm{B} 1-\mathrm{Al}$ 键 (574 GPa)。Cr-B 键的强度与它们在 $\mathrm{Cr}-\mathrm{B}$ 结构单 元中的位置有关: 在层的边缘, $\mathrm{Cr} 1$ 原子采用与 $\mathrm{CrB}$ 中相似的结构, 更接近于六个相邻的 $\mathrm{B}$ 原子。 $\mathrm{Cr} 1-\mathrm{B} 2$ 具有与 $\mathrm{CrB}$ 中相似的刚性, 并且是 $\mathrm{Cr}_{4} \mathrm{AlB}_{4}$ 中刚度最大的键; 朝向 $\mathrm{Cr}-\mathrm{B}$ 平板的中心位置, $\mathrm{Cr}-\mathrm{B}$
键变得更软。

为了进一步深入理解 $\mathrm{Cr}_{4} \mathrm{AlB}_{4}$ 的化学键合, 本 工作研究了它的态密度, 电荷密度分布和电荷转移 情况，如图 4、图 5 和表 4 所示。通过图 4 中 $\mathrm{Cr}_{4} \mathrm{AlB}_{4}$ 的原子分波态密度可以看出在能级较低处 $(-13 \sim$ $-9 \mathrm{eV})$, 主要由 B1/2-2s 态组成，表明 B-B 共价键的 高强度。在较高能量下, $\mathrm{B}-2 \mathrm{p}$ 和 $\mathrm{Cr}-3 \mathrm{~d}$ 的分波态密 度在 $-6 \mathrm{eV}$ 至 $-3.5 \mathrm{eV}$ 的区域重叠，具有重合峰，表 明这些轨道之间强烈的杂化。杂化区域的宽能量范 围和高态密度则意味着相对较强的 $\mathrm{Cr}-\mathrm{B}$ 共价键。 在 $-3.5 \mathrm{eV}$ 和 $-2 \mathrm{eV}$ 之间, Al-p、B1-p 和 Cr1-p 轨道之 间还有一个重叠区域，这意味着在 $\mathrm{Al}$ 和 $\mathrm{B} 1$ 之间及 $\mathrm{Al}$ 和 $\mathrm{Cr} 1$ 具有弱的化学键。类似的, $\mathrm{CrB}$ 在 $-6 \mathrm{eV}$ 至 $-3 \mathrm{eV}$ 区域 B- $2 \mathrm{p}$ 和 $\mathrm{Cr}-3 \mathrm{~d}$ 轨道之间发成重叠, 意味 着相对较强的 $\mathrm{Cr}-\mathrm{B}$ 共价键。可见, DOS 的分析结果 与键刚度模型的分析结果一致。

通过图 5 可以发现 $\mathrm{B}$ 原子与 $\mathrm{B}$ 原子之间存在明 显的电荷堆积现象，表明 B-B 键为较强的共价键。 而 $\mathrm{Cr}$ 原子与 $\mathrm{B}$ 原子之间的电荷堆积情况却不完全 相同，其中 $\mathrm{Cr} 1$ 原子与 $\mathrm{B} 2$ 原子，及 $\mathrm{Cr} 2$ 原子与

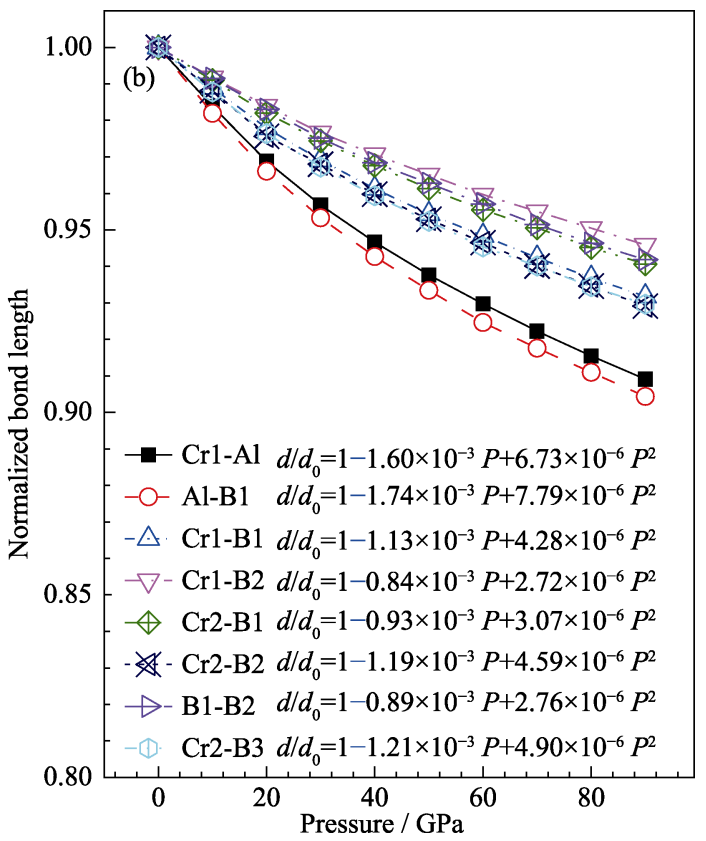

图 3 压力对 $\mathrm{Cr}_{4} \mathrm{AlB}_{4}$ 的归一化体积 $V / V_{0}$ 及归一化晶格常数 $a / a_{0}, b / b_{0}$ 和 $c / c_{0}$ (a) 和归一化键长 $d / d_{0}$ (b)的影响

Fig. 3 Pressure dependence of the normalized cell volume $V / V_{0}$ as well as the normalized lattice parameters $a / a_{0}, b / b_{0}$ and $c / c_{0}$ of $\mathrm{Cr}_{4} \mathrm{AlB}_{4}$ (a) and the normalized bond length $d / d_{0}$ in $\mathrm{Cr}_{4} \mathrm{AlB}_{4}$ (b) 

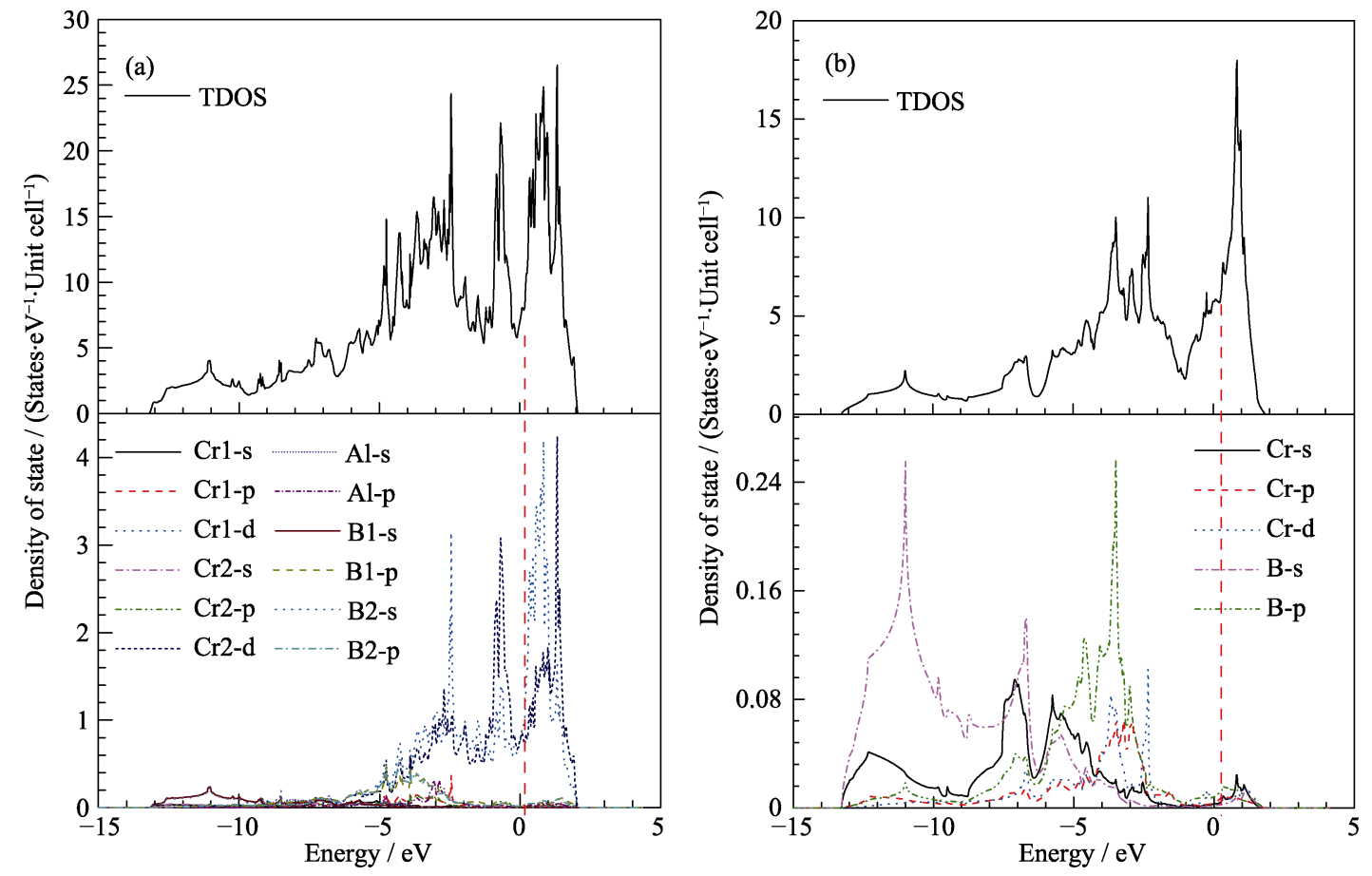

图 $4 \mathrm{Cr}_{4} \mathrm{AlB}_{4}(\mathrm{a})$ 和 $\mathrm{CrB}(\mathrm{b})$ 的态密度及分波态密度

Fig. 4 Total and partial density of states of $\mathrm{Cr}_{4} \mathrm{AlB}_{4}$ (a) and $\mathrm{CrB}$ (b)

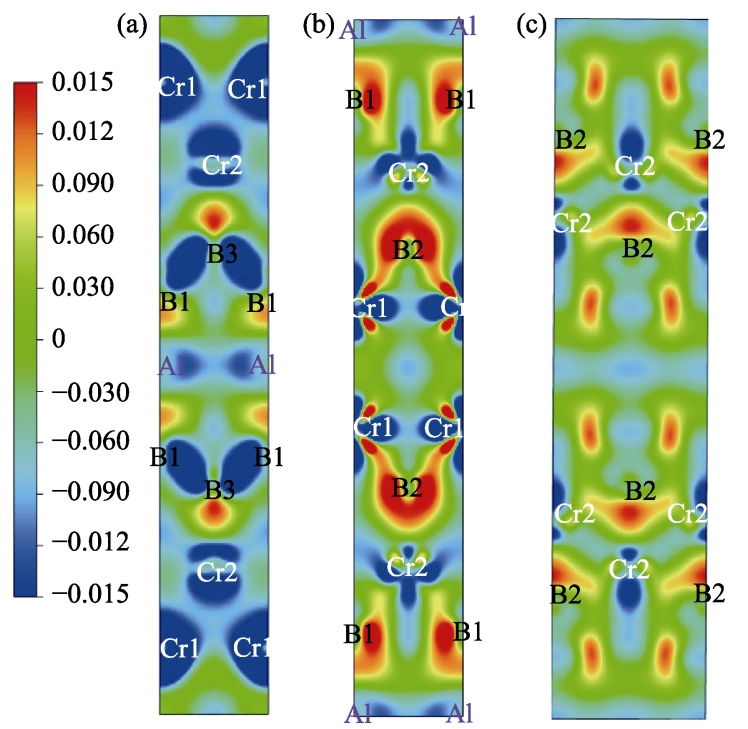

图 $5 \mathrm{Cr}_{4} \mathrm{AlB}_{4}$ 在 $(001)(\mathrm{a}) 、(100)(\mathrm{b})$ 和(101) (c)平面上的差分 电荷密度

Fig. 5 Electron density difference maps on (001) (a), (100) (b) and (101) (c) planes of $\mathrm{Cr}_{4} \mathrm{AlB}_{4}$

表 $4 \mathrm{Cr}_{4} \mathrm{AlB}_{4}, \mathrm{Cr}_{2} \mathrm{AlB}_{2}$ 和 $\mathrm{CrB}$ 的贝德电荷分析 Table 4 Bader charge analysis of $\mathrm{Cr}_{4} \mathrm{AlB}_{4}, \mathrm{Cr}_{2} \mathrm{AlB}_{2}$ and $\mathrm{CrB}$

\begin{tabular}{lccccc}
\hline Boder charge/e & $\mathrm{Cr} 1$ & $\mathrm{Cr} 2$ & $\mathrm{~A} 1$ & $\mathrm{~B} 1$ & $\mathrm{~B} 2$ \\
\hline $\mathrm{CrB}$ & -0.79 & - & - & 0.78 & - \\
$\mathrm{Cr}_{2} \mathrm{AlB}_{2}{ }^{[26]}$ & -0.37 & - & -1.50 & 1.12 & - \\
$\mathrm{Cr}_{4} \mathrm{AlB}_{4}$ & -0.39 & -0.76 & -1.48 & 1.15 & 0.74 \\
\hline
\end{tabular}

$\mathrm{B} 1$ 原子之间电荷堆积最为强烈, 而 $\mathrm{Cr} 2$ 原子与 $\mathrm{B} 3$ 原子之间电荷堆积情况最弱, 这与 $\mathrm{Cr}-\mathrm{B}$ 键刚度计
算结果(表 2)一致。对 $\mathrm{Cr}_{4} \mathrm{AlB}_{4}$ 的 Bader 电荷分析表 明在 $\mathrm{Cr}-\mathrm{B}$ 层层间电荷转移情况与 $\mathrm{CrB}$ 相似, $\mathrm{Cr}$ 原 子失去 0.76 个电子而 $\mathrm{B}$ 原子得到 0.74 个电子; 而在 $\mathrm{Cr}-\mathrm{B}$ 层边缘，由于 $\mathrm{Al}$ 原子的插入形成 $\mathrm{Cr}-\mathrm{Al}$ 键和 $\mathrm{B}-\mathrm{Al}$ 键, $\mathrm{Cr}$ 原子失去电子变少, 减为 0.39 个, $\mathrm{B}$ 原 子得到电子变多, 增加到 1.15 个, 其中 $\mathrm{Al}$ 原子失去 1.48 个电子用于形成 $\mathrm{Cr}-\mathrm{Al}$ 键和 $\mathrm{B}-\mathrm{Al}$ 键(见表 4)。

对 MAX 相的研究表明较弱的 M-A 键在其非凡 力学行为中扮演了关键角色, 如高断裂韧性和损伤 容限、低硬度、微尺度塑性变形等 ${ }^{[31]}$ 。采用键刚度 模型研究表明, 对包括 MAX 和 MAB 相的三元层状 化合物陶瓷来说, 最弱化学键刚度与最强化学键刚 度的比值与其宏观力学行为密切相关 ${ }^{[25]}$ : 当该比值 大于 $1 / 2$ 时, 这类陶瓷材料表现出典型脆性陶瓷的 低损伤容限和断裂行为, 如三元碳化物 $(\mathrm{MC})_{n} \mathrm{Al}_{3} \mathrm{C}_{2}$ 和 $(\mathrm{MC}){ }_{n} \mathrm{Al}_{4} \mathrm{C}_{3}$ 陶瓷 ${ }^{[32]}$; 而当该比值小于 $1 / 2$ 时, 这 类陶瓷则表现出高损伤容限和高断裂韧性, 如 MoAlB 和典型的 MAX 相陶瓷 ${ }^{[33-34]}$ 。

如表 2 所示, $\mathrm{Cr}_{4} \mathrm{AlB}_{4}$ 中刚度最低的键为 $\mathrm{B} 1-\mathrm{Al}$ 键和 $\mathrm{Cr}-\mathrm{Al}$ 键, 与 $\mathrm{MAX}$ 相的 $\mathrm{M}-\mathrm{A}$ 键相似。其中 $\mathrm{B} 1-\mathrm{Al}$ 键 (574 GPa) 和 $\mathrm{Cr} 1-\mathrm{Al}$ 键刚度 (625 GPa)分别 是最强的 $\mathrm{Cr} 1-\mathrm{B} 2$ 键刚度 $(1190 \mathrm{GPa})$ 的 0.48 和 0.52 倍。因此可以预见, MAX 相中存在的反常力学行为 也应该存在于 $\mathrm{Cr}_{4} \mathrm{AlB}_{4}$ 中。最近对 MoAlB 的理论工 作也得出了类似的结论 ${ }^{[25]}$, 并且与在实验观察到的 高损伤容限一致 ${ }^{[7]}$ 。 
表 $5 \mathrm{Cr}_{4} \mathrm{AlB}_{4}$ 的二阶弹性常数 $\left(c_{i j} / \mathrm{GPa}\right)$ 、体积模量 $(B / G P a)$ 、剪切模量 $(G / G P a)$ 、杨氏模量 $(E / G P a) 、$ 泊松比 $(\mu)$ 和德拜温度 $\left(\theta_{\mathrm{D}} / \mathrm{K}\right)$

Table 5 The calculated second-order elastic constants $\left(c_{\mathrm{ij}} / \mathrm{GPa}\right)$, shear moduli $(G / \mathrm{GPa})$, bulk moduli $(B / G P a)$, Young's moduli (E/GPa), Poisson ratio $(\mu)$, and Debye temperature $\left(\theta_{\mathrm{D}} / \mathrm{K}\right)$ of $\mathrm{Cr}_{4} \mathrm{AlB}_{4}$

\begin{tabular}{cccccccccccccccc}
\hline Compound & $c_{11}$ & $c_{12}$ & $c_{13}$ & $c_{22}$ & $c_{23}$ & $c_{33}$ & $c_{44}$ & $c_{55}$ & $c_{66}$ & $G$ & $B$ & $G / B$ & $E$ & $\mu$ & $\theta_{\mathrm{D}} / \mathrm{K}$ \\
\hline $\mathrm{Cr}_{4} \mathrm{AlB}_{4}$ & 594 & 112 & 126 & 472 & 120 & 467 & 185 & 176 & 231 & 194 & 245 & 0.79 & 460 & 0.19 & 923 \\
$\mathrm{Cr}_{2} \mathrm{AlB}_{2}{ }^{[26]}$ & 505 & 101 & 111 & 410 & 109 & 437 & 158 & 162 & 209 & 174 & 221 & 0.79 & 414 & 0.19 & 892 \\
$\mathrm{MoAlB}^{[25]}$ & 327 & 143 & 150 & 313 & 128 & 384 & 187 & 156 & 166 & 142 & 207 & 0.66 & 347 & 0.22 & 675 \\
$\mathrm{CrB}$ & 495 & 165 & 165 & 581 & 147 & 579 & 220 & 220 & 265 & 219 & 289 & 0.78 & 525 & 0.2 & 963 \\
$\mathrm{Cr}_{2} \mathrm{AlC}^{[23]}$ & 396 & 117 & 156 & - & - & 382 & 173 & - & - & 146.9 & 225.8 & - & 357.7 & 0.236 & 774 \\
\hline
\end{tabular}

\section{4 弹性性能}

晶体的弹性刚度决定了其对平衡位置附近的应 变(或应力)的响应, 表 5 为 $\mathrm{Cr}_{4} \mathrm{AlB}_{4}$ 的二阶弹性常数 及工程弹性性能, 并将其与 $\mathrm{MAB}$ 相 $\mathrm{Cr}_{2} \mathrm{AlB}_{2}$ 、 MoAlB，二元喼化物 $\mathrm{CrB}$ 和 MAX 相化合物 $\mathrm{Cr}_{2} \mathrm{AlC}$ 进行对比。 $c_{11} 、 c_{22} 、 c_{33}$ 分别表示对主应变 $\varepsilon_{11} 、 \varepsilon_{22}$ 和 $\varepsilon_{33}$ 具有的正变形阻力。 $c_{11}$ 具有最大值 $(594 \mathrm{GPa})$, $c_{22}$ 具有最小值 $(472 \mathrm{GPa})$ 。这与可压缩性的研究结果 一致(图 3)。其原因是由于 B-B 键的高刚性, 具有最 大弹性常数方向应平行于 $\mathrm{B}-\mathrm{B}$ 锯齿形/六角形链, 对应 $\mathrm{Cr}_{4} \mathrm{AlB}_{4}$ 的 $a$ 轴方向。

同 $\mathrm{CrB}$ 相比, $\mathrm{Cr}_{4} \mathrm{AlB}_{4}$ 的 $c_{11}$ 略大而 $c_{22}, c_{33}$ 略小, 其原因是 $\mathrm{Cr}_{4} \mathrm{AlB}_{4}$ 的 $a$ 轴方向为较强的 $\mathrm{B}-\mathrm{B}$ 共价键 $(1123 \mathrm{GPa})$ 折叠方向, 而 $\mathrm{CrB}$ 为 $c$ 轴方向。若选取相 同方向, $\mathrm{CrB}$ 的二阶弹性常数大于 $\mathrm{Cr}_{4} \mathrm{AlB}_{4}$, 因为较 弱的 $\mathrm{Cr}-\mathrm{Al}$ 键 $(625 \mathrm{GPa})$ 和 $\mathrm{B}-\mathrm{Al}$ 键 $(574 \mathrm{GPa})$ 使其弹 性常数减小。 $\mathrm{Cr}_{4} \mathrm{AlB}_{4}$ 的剪切变形值 $c_{44} 、 c_{55} 、 c_{66}$ 与 $\mathrm{CrB}$ 相比也略有减小。与 $\mathrm{MAB}$ 相的 $\mathrm{Cr}_{2} \mathrm{AlB}_{2}$ 相比, $\mathrm{Cr}_{4} \mathrm{AlB}_{4}$ 的二阶弹性常数较大, $\mathrm{Cr}_{2} \mathrm{AlB}_{2}$ 为单层的 $\mathrm{Cr}-\mathrm{B}$ 层, 而 $\mathrm{Cr}_{4} \mathrm{AlB}_{4}$ 为双层, $\mathrm{Cr}-\mathrm{B}$ 层具有较高的刚度。

表 5 还列出了 $\mathrm{Cr}_{4} \mathrm{AlB}_{4}$ 的工程弹性常数, 包括泊 松比 $(\mu)$ 和体积模量 $(B)$, 杨氏模量 $(E)$ 和剪切模量 $(G)$, 根据弹性常数 $c_{\mathrm{ij}}$ 计算。 $\mathrm{Cr}_{4} \mathrm{AlB}_{4}$ 的体积模量 $(B)$, 杨 氏模量 $(E)$ 和剪切模量 $(G)$ 高于同为 $\mathrm{MAB}$ 相的 $\mathrm{Cr}_{2} \mathrm{AlB}_{2}$ 和 $\mathrm{MoAlB}$, 但低于二元嗍化物 $\mathrm{CrB}$ 。此外, $\mathrm{Cr}_{4} \mathrm{AlB}_{4}$ 与 $\mathrm{Cr}_{2} \mathrm{AlB}_{2}$ 及 $\mathrm{CrB}$ 有着相近的泊松比, $\mathrm{Cr}_{2} \mathrm{AlC}$ 明显高于三者。 $G / B$ 比通常用于描述材料的 延展性或脆性, 低 $G / B$ 表示较好的延展性, 根据 Pugh 标准划分临界值为 $0.57^{[35]}$ 。 $\mathrm{Cr}_{4} \mathrm{AlB}_{4}$ 的 $G / B$ 比 值为 0.79 , 大于 0.57 表明其属于脆性材料, 硬度较 高。但对大部分三元层状化合物 MAX 相及 MAB 相的分析并不可靠, 例如对 MoAlB 的分析中, 其 $G / B$ 较高但仍具有较好的损伤容限 ${ }^{[25]}$ 。随着 $\mathrm{Al}$ 原子 的插入, 德拜温度显著降低。德拜温度对应于晶格 振动的最高频率, 这实际上是晶体内键合强弱的反 映。这表明 $\mathrm{Cr}_{4} \mathrm{AlB}_{4}$ 中的平均键合应弱于 $\mathrm{CrB}$, 并具 有较大的热膨胀。

\section{3 结论}

本研究采用基于第一性原理的“线性优化法”和 “键刚度”理论模型分别研究了 $\mathrm{Cr}_{4} \mathrm{AlB}_{4}$ 的物相稳定 性和力学行为。

1) $\mathrm{Cr}_{4} \mathrm{AlB}_{4}$ 在声子谱中并未出现虚频, 表明其 具有本征稳定性。且高频的声子频率几乎被 $\mathrm{B}$ 原子 占据, 而 $\mathrm{Cr}$ 和 $\mathrm{Al}$ 声子态的频率总是在较低位置。

2) $\mathrm{Cr}_{4} \mathrm{AlB}_{4}$ 与其他 $\mathrm{Cr}-\mathrm{Al}-\mathrm{B}$ 系竞争相相比, 具有 最低能量, 处于热力学稳定状态。

3) 较强的 B-B 共价键导致了 $\mathrm{Cr}_{4} \mathrm{AlB}_{4}$ 可压缩行 为的各向异性, 其中 $a$ 轴的抗压能力最好, $c$ 轴的抗 压能力最差。

4) $\mathrm{Cr}_{4} \mathrm{AlB}_{4}$ 中刚度最低的键为最强键的 0.48 倍, 因此本文预测 $\mathrm{Cr}_{4} \mathrm{AlB}_{4}$ 中也具有类似 $\mathrm{MAX}$ 相的高 损伤容限和断裂韧性。

5) 随着 $\mathrm{Al}$ 原子插入 $\mathrm{CrB}$ 形成 $\mathrm{Cr}_{4} \mathrm{AlB}_{4}$, 弹性常 数略有降低。德拜温度显著降低则表明晶体内键合 的减弱。

\section{参考文献:}

[1] FAHRENHOLtZ W G, HiLMAS G E, TALMY I G, et al. Refractory diborides of zirconium and hafnium. J. Am. Ceram. Soc., 2007, 90(5): 1347-1364.

[2] BARSOUM M W. The $\mathrm{M}_{N+1} \mathrm{AX}_{N}$ phases: a new class of solids; thermodynamically stable nanolaminates. Prog. Solid State Chem., 2000, 28(1): 201-281.

[3] ADE M, HILLEBRECHT H. Ternary borides $\mathrm{Cr}_{2} \mathrm{AlB}_{2}, \mathrm{Cr}_{3} \mathrm{AlB}_{4}$, and $\mathrm{Cr}_{4} \mathrm{AlB}_{6}$ : the first members of the series $\left(\mathrm{CrB}_{2}\right)_{n} \mathrm{CrAl}$ with $n=$ $1,2,3$ and a unifying concept for ternary borides as MAB-phases. Inorganic Chemistry, 2015, 54(13): 6122-6135.

[4] JEITSCHKO W. The crystal structure of $\mathrm{Fe}_{2} \mathrm{AlB}_{2}$. Acta Crystallographica, 1969, 25(1): 163-165.

[5] NOWOTNY H, ROGL P. Ternary Metal Borides. Berlin Heidelberg: Springer. 1977: 413-438.

[6] TAN XIAOYAN, CHAI PING, THOMPSON COREY M, et al. Magnetocaloric effect in $\mathrm{AlFe}_{2} \mathrm{~B}_{2}$ : toward magnetic refrigerants from earth-abundant elements. Journal of the American Chemical Society, 2013, 135(25): 9553-9557.

[7] KOTA S, ZAPATA-SOLVAS E, LY A, et al. Synthesis and charac- 
terization of an alumina forming nanolaminated boride: MoAlB. Sci. Rep., 2016, 6: 26475.

[8] LI NING, BAI YUELEI, WANG SHUAI, et al. Rapid synthesis, electrical, and mechanical properties of polycrystalline $\mathrm{Fe}_{2} \mathrm{AlB}_{2}$ bulk from elemental powders. Journal of the American Ceramic Society, 2017, 100(10): 4407-4411.

[9] KADAS K, IUSAN D, HELLSVIK J, et al. $\mathrm{AlM}_{2} \mathrm{~B}_{2}(\mathrm{M}=\mathrm{Cr}, \mathrm{Mn}$, $\mathrm{Fe}, \mathrm{Co}, \mathrm{Ni})$ : a group of nanolaminated materials. Journal of Physics-Condensed Matter, 2017, 29(15): 155402.

[10] ZHOU YANCHUN, XIANG HUIMIN, DAI FUZHI, et al. Electrical conductive and damage-tolerant nanolaminated MAB phases $\mathrm{Cr}_{2} \mathrm{AlB}_{2}, \mathrm{Cr}_{3} \mathrm{AlB}_{4}$ and $\mathrm{Cr}_{4} \mathrm{AlB}_{6}$. Materials Research Letters, 2017, 5(6): $1-9$.

[11] DAI FUZHI, FENG ZHIHAI, ZHOU YANCHUN. Easily tiltable B Al B linear chain: the origin of unusual mechanical properties of nanolaminated MAB phases $\left(\mathrm{CrB}_{2}\right)_{n} \mathrm{CrAl}$. Journal of Alloys and Compounds, 2017, 723: 462-466.

[12] CHABAN N F, KUZ'MA YU B. Cheminform abstract: Ternaere systeme Cr-Al-B und Mn-Al-B. Chemischer Informationsdienst, 1974, DOI: 10.1002/chin.197404029.

[13] ZHANG HAIMING, DAI FU-ZHI, XIANG HUIMIN, et al. Crystal structure of $\mathrm{Cr}_{4} \mathrm{AlB}_{4}$ : a new MAB phase compound discovered in Cr-Al-B system. Journal of Materials Science \& Technology, 2019, 35(4): 530-534.

[14] DAI FUZHI, ZHANG HAIMING, XIANG HUIMIN, et al. Theoretical investigation on the stability, mechanical and thermal properties of the newly discovered MAB phase $\mathrm{Cr}_{4} \mathrm{AlB}_{4}$. Journal of Materials Science \& Technology, 2019, DOI: 10.1016/j.jmst.2019.01.017.

[15] LIU BIN, LIU YUCHEN, ZHU CHANGHUA, et al. Advances on strategies for searching for next generation thermal barrier coating materials. Journal of Materials Science \& Technology, 2019, 35(5): 833-851.

[16] LIU YUCHEN, COOPER VALENTINO R, WANG BANGHUI, et al. Discovery of $\mathrm{ABO}_{3}$ perovskites as thermal barrier coatings through high-throughput first principles calculations. Materials Research Letters, 2019, 7(4): 145-151.

[17] KRESSE G, FURTHMÜLLER J. Efficient iterative schemes for $a b$ initio total-energy calculations using a plane-wave basis set. Phys. Rev. B: Condens. Matter, 1996, 54(16): 11169-11186.

[18] PERDEW J P, BURKE K, ERNZERHOF M. Generalized gradient approximation made simple. Physical Review Letters, 1996, 77(18): 3865-3868.

[19] HAMMER B, HANSEN L B, NORSKOV J K. Improved adsorption energetics within density-functional theory using revised PerdewBurke-Ernzerhof functionals. Phys. Rev. B, 1999, 59(11): 7413-7421.

[20] PERDEW JOHN P, CHEVARY J A, VOSKO S H, et al. Atoms, molecules, solids, and surfaces: applications of the generalized gradient approximation for exchange and correlation. Phys. Rev. B:
Condens. Matter, 1992, 46(11): 6671-6687.

[21] DAHLQVIST M, ALLING B, ROSÉN J. Stability trends of MAX phases from first principles. Physical Review B, 2010, 81(22): 220102.

[22] BAI YUELEI, HE XIAODONG, SUN YUE, et al. Chemical bonding and elastic properties of $\mathrm{Ti}_{3} \mathrm{AC}_{2}$ phases $(\mathrm{A}=\mathrm{Si}, \mathrm{Ge}$, and Sn): a first-principle study. Solid State Sciences, 2010, 12(7): $1220-1225$.

[23] SUN ZHIMEI, LI SA, AHUJA RAJEEV, et al. Calculated elastic properties of $\mathrm{M}_{2} \mathrm{AlC}(\mathrm{M}=\mathrm{Ti}, \mathrm{V}, \mathrm{Cr}, \mathrm{Nb}$ and Ta). Solid State Communications, 2004, 129(9): 589-592.

[24] ANDERSON ORSON L. A simplified method for calculating the debye temperature from elastic constants. J. Phys. Chem. Solids, 1963, 24(7): 909-917.

[25] BAI YUELEI, QI XINXIN, DUFF ANDREW, et al. Density functional theory insights into ternary layered boride MoAlB. Acta Materialia, 2017, 132: 69-81.

[26] BAI YUELEI, QI XINXIN, HE XIAODONG, et al. Phase stability and weak metallic bonding within ternary-layered borides CrAlB, $\mathrm{Cr}_{2} \mathrm{AlB}_{2}, \mathrm{Cr}_{3} \mathrm{AlB}_{4}$, and $\mathrm{Cr}_{4} \mathrm{AlB}_{6}$. Journal of the American Ceramic Society, 2019, 102(6): 3715-3727.

[27] CHONG XIAOYU, JIANG YEHUA, ZHOU RONG, et al. Elastic properties and electronic structures of $\mathrm{Cr}_{x} \mathrm{~B}_{y}$ as superhard compounds. Journal of Alloys and Compounds, 2014, 610: 684-694.

[28] ONODERA A, HIRANO H, YUASA T, et al. Static compression of $\mathrm{Ti}_{3} \mathrm{SiC}_{2}$ to $61 \mathrm{GPa}$. Appl. Phys. Lett., 1999, 74(25): 3782-3784.

[29] WANG JIEMIN, ZHOU YANCHUN. Ab initio investigation of the electronic structure and bonding properties of the layered ternary compound $\mathrm{Ti}_{3} \mathrm{SiC}_{2}$ at high pressure. Journal of Physics-Condensed Matter, 2003, 15(12): 1983-1991.

[30] FRANCIS BIRCH. Finite elastic strain of cubic crystals. Physical Review, 1947, 71(11): 809-824.

[31] BARSOUM W MICHEL. Mechanical Properties: Ambient Temperature. MAX Phases: Properties of Machinable Ternary Carbides and Nitrides. Weinheim: Wiley, 2013.

[32] BAI YUELEI, DUFF ANDREW, JAYASEELAN DANIEL DONI, et al. DFT predictions of crystal structure, electronic structure, compressibility, and elastic properties of Hf-Al-C carbides. Journal of the American Ceramic Society, 2016, 99(10): 3449-3457.

[33] BAI YUELEI, HE XIAODONG, WANG RONGGUO, et al. An $a b$ initio study on compressibility of Al-containing MAX-phase carbides. Journal of Applied Physics, 2013, 114(17): 173709.

[34] WANG CHANGYING, HAN HAN, ZHAO YUANYUAN, et al. Elastic, mechanical, electronic, and defective properties of $\mathrm{Zr}-\mathrm{Al}-\mathrm{C}$ nanolaminates from first principles. Journal of the American Ceramic Society, 2018, 101(2): 756-772.

[35] PUGH S F. Relations between the elastic moduli and the plastic properties of polycrystalline pure metals. Philosophical Magazine, 2009, 45(367): 823-843. 